\title{
Transient cavitation flow of the neat PODE (polyoxymethylene dimethyl ether) within a solenoid injector
}

\author{
Chuqiao Wang*1, Andreas Röll',3, Konstantinos Kolovos ${ }^{4}$, Alvaro Vidal Roncero ${ }^{4}$, Yu Sun², \\ Phoevos Koukouvinis ${ }^{4}$, Manolis Gavaises ${ }^{4}$, Markus Brautsch ${ }^{3}$, Raphael Lechner ${ }^{3}$, \\ Nicholas O'Connell ${ }^{3}$, Zhixia $\mathrm{He}^{1}$ and Fuqiang Luo ${ }^{2}$ \\ ${ }^{1}$ School of Energy and Power Engineering, Jiangsu University, China \\ 2 School of Automotive and Traffic Engineering, Jiangsu University, China \\ ${ }^{3}$ Institute of Energy Technology at OTH Technical University of Applied Sciences, Germany \\ ${ }^{4}$ School of Mathematics, Computer Science and Engineering, City, University of London, UK \\ ${ }^{*}$ Corresponding author email: cqwang@ujs.edu.cn
}

\begin{abstract}
The impact of transient needle moving on the flow characteristics within a solenoid injector using the neat PODE (polyoxymethylene dimethyl ether) was investigated in this work. An explicit density-based solver suitable for multiphase flows was implemented into OpenFOAM ${ }^{\circledR}$ to solve the compressible Navier-Stokes equations of the Arbitrary Lagrangian-Eulerian (ALE) formulation, while the turbulence is resolved using a RNG $\mathrm{k}-\varepsilon$ model with the Reboud correction. Phase change between the liquid and vapor phases was predicted by the variation in the density utilizing the homogeneous mixture model. A barotropic equation of state (EoS), i.e. the density as a function of pressure, where thermal effects and saturation properties are simplified at a constant temperature, is adopted to resolve the thermodynamic model. Numerical simulation was carried out on a double-layer multi-hole diesel injector during ballistic condition. The results show that cavitation formed on the upper side of both orifices, which is attributed to the flow acceleration in the narrow needle seat area, changing the flow direction significantly before passing through the two orifices heading to different orientations. Keywords

PODE, cavitation, needle movement, HEM, OpenFOAM
\end{abstract}

\section{Introduction}

Transient flow characteristics of the neat PODE (polyoxymethylene dimethyl ether) within the diesel injector are complex, with high velocity magnitude $(\sim 700 \mathrm{~m} / \mathrm{s})$ in a small geometric scale $(\sim 1 \mathrm{~mm})$. Besides, the cavitation phenomena will be induced due to the pressure drop and flow direction change. A large amount of numerical and experimental study was conducted based on the incompressible URANS method and cavitation visualization techniques[1]. Detailed cavitating flow within the nozzle such as geometric induced cavitation and string cavitation have been noticed from both simulation and experiments[2]. However, it seems that previous study was mainly conducted by an incompressible assumption, which is not very accurate at real working conditions, especially at high pressures[3]. Furthermore, the number of orifices increases with the growth of the injection pressure, which introduces a double-layer geometry to make the spray jets more uniform than the one-layer nozzle[4]. In this study, an explicit density-based solver of the compressible Navier-Stokes equations suitable for cavitation simulation was utilised to capture the vortex structures and their strong correlation with the formation process of vapor[5]. The transient moving of needle was resolved by a cell-based cell deformation approach, and the phase change was predicted by the variation of the density using the barotropic equation of state (EoS)[6], [7].

\section{Numerical modelling}




\section{Mathematical model}

The modified in-house code based on the explicit density based solver has been implemented into OpenFOAM® to solve the compressible form of the Navier-Stokes equations. The energy conservative equation has been neglected in this study by adopting an alternative approach, the barotropic equation of the state (EoS). The continuity equation and momentum equation employed for the compressible flow of RANS simulations are expressed as:

$$
\begin{aligned}
& \frac{\partial \rho}{\partial t}+\nabla \cdot(\rho \boldsymbol{U})=0 \\
& \frac{\partial(\rho \boldsymbol{U})}{\partial t}+\nabla \cdot(\rho \boldsymbol{U} \boldsymbol{U})=-\nabla p+\nabla \cdot \tau-\nabla \cdot(\rho \boldsymbol{R})
\end{aligned}
$$

where $\rho$ is the density of the mixture phase, $\boldsymbol{U}$ is the velocity vector, $p$ is the pressure, $\tau$ is the stress tensor, and $R$ represents the Reynold stresses following the Boussinesq assumption:

$$
\begin{aligned}
& \frac{\partial \rho}{\partial t}+\nabla \cdot(\rho \boldsymbol{U})=0 \\
& \frac{\partial(\rho \boldsymbol{U})}{\partial t}+\nabla \cdot(\rho \boldsymbol{U} \boldsymbol{U})=-\nabla p+\nabla \cdot \tau-\nabla \cdot(\rho \boldsymbol{R})
\end{aligned}
$$

where $\mu$ is the viscosity of the mixture phase and $l$ is the identify tensor.

The turbulence within the injector is resolved by a modified RNG k- $\varepsilon$ model with the Reboud correction, taking into account the compressible effects which could capture the self-oscillation behavior of cavitating flow[8], [9]. The correction is introduced by reducing the eddy viscosity for the mixture phase, expressed as:

$$
\mu_{\tau}=\frac{\rho C_{\mu} k^{2}}{\varepsilon}
$$

where $k$ is the turbulent kinetic energy, $\varepsilon$ is the dissipation rate, $C_{\mu}=0.085$ and $\rho(\alpha)$ is expressed as:

$$
\rho(\alpha)=\rho_{v}+(1-\alpha)^{n}\left(\rho_{l}-\rho_{v}\right)
$$

where $\alpha$ is the volume fraction; the subscript $I$ and $v$ indicate the liquid and vapor phase respectively, and $n$ is a constant taking the value at $\sim 10$.

Cavitation model

The HEM, i.e. homogeneous equilibrium model was utilized in this study to resolve the phase change. For the HEM, each phase shares the same pressure and temperature, and the phase change between the liquid and the liquid-vapor mixture is predicted by the corresponding EoS. The barotropic EoS, as one of the most simplified approach in HEM, which strongly links the density variations to the pressure evolution was adopted[10].

The modified Tait EoS was utilised for the liquid phase, while the isentropic-resembling process was employed for the liquid-vapour mixture:

$$
p(\rho)=\left\{\begin{array}{cl}
\left(B+p_{\text {sat }}\right)\left[\left(\frac{\rho}{\rho_{\text {sat }, l}}\right)^{n}\right]-B, & \rho \geq \rho_{\text {sat }, L} \\
p_{\text {sat }}+C\left[\frac{1}{\rho_{\text {sat }, l}}-\frac{1}{\rho}\right], & \rho<\rho_{\text {sat }, L}
\end{array}\right.
$$


where $B$ is the bulk modulus of liquid, exponent $n$ represents the stiffness which is usually set as 7.15 for the weakly compressible liquid[9], [11], $p_{\text {sat }}$ is the saturation pressure of the liquid and $\rho_{s a t, l}$ is the density of the liquid at saturation pressure.

The thermophysical properties of the neat PODE was measured by Flucon Fluid Control $\mathrm{GmbH}$ at the temperature of $323 \mathrm{~K}$, while the vapour pressure was calculated from the Antoine equation, presented in the Table 1[12].

Table 1 Thermophysical properties

\begin{tabular}{|c|c|c|c|c|c|}
\hline \multicolumn{3}{|c|}{ Liquid properties } & \multicolumn{3}{c|}{ Vapour properties } \\
\hline \multicolumn{1}{|c|}{} & 170.864 & $\mathrm{MPa}$ & & & \\
\hline$p_{\text {sat }}$ & 1000 & $\mathrm{~Pa}$ & & & \\
\hline$\rho_{\text {sat }, l}$ & 1029.45 & $\mathrm{~kg} / \mathrm{m}^{3}$ & $\rho_{\text {sat }, v}$ & 0.1 & $\mathrm{~kg} / \mathrm{m}^{3}$ \\
\hline$c_{\text {sat }, l}$ & 1055.29 & $\mathrm{~m} / \mathrm{s}$ & $c_{\text {sat }, v}$ & 225 & $\mathrm{~m} / \mathrm{s}$ \\
\hline$v_{\text {sat }, l}$ & $1.1 * 10^{-6}$ & $\mathrm{~m}^{2} / \mathrm{s}$ & $v_{\text {sat }, v}$ & $4 * 10^{-5}$ & $\mathrm{~m}^{2} / \mathrm{s}$ \\
\hline
\end{tabular}

Geometry model and mesh topology

An eight-hole double layer solenoid injector was used in this study. In view of the computational cost, a quarter of the injector was adopted as the numerical geometry. Table 2 presents the detailed geometric parameters of the quarter model in terms of the hole lengths, inlet rounding corners, inlet and outlet diameters. The lower layered orifice is specified as orifice 1 , while the upper layered hole as 2.

Table 2 Geometric parameters

\begin{tabular}{ccc}
\hline Nozzle holes & Lower layered & Upper layered \\
\hline Orifice inlet diameter $/ \mu \mathrm{m}$ & 180.2 & 180.1 \\
Orifice outlet diameter $/ \mu \mathrm{m}$ & 180.2 & 180.2 \\
Rounding diameter $/ \mu \mathrm{m}$ & 31 & 32 \\
Orifice length $/ \mathrm{mm}$ & 0.65 & 0.65 \\
\hline
\end{tabular}

Figure 1 represents the boundary naming of the model. The numerical model was discretized into $\sim 400,000$ hexahedral cells. In order to capture the transient cavitating flow within the nozzle, the mesh resolution was increased in critical areas, such as nozzle hole inlet and the needle seat. With the mean fuel flow velocity being around $500 \mathrm{~m} / \mathrm{s}$, an estimation of the Taylor length scale yielded around $2.1 \mu \mathrm{m}$. The inlet pressure and outlet pressure were set as 150 $\mathrm{MPa}$ and $5 \mathrm{MPa}$ respectively. The needle moving was represented by a cell-based mesh deformation method uniformly, and the time step was 0.2 ns corresponds to the CFL number of 0.7 .

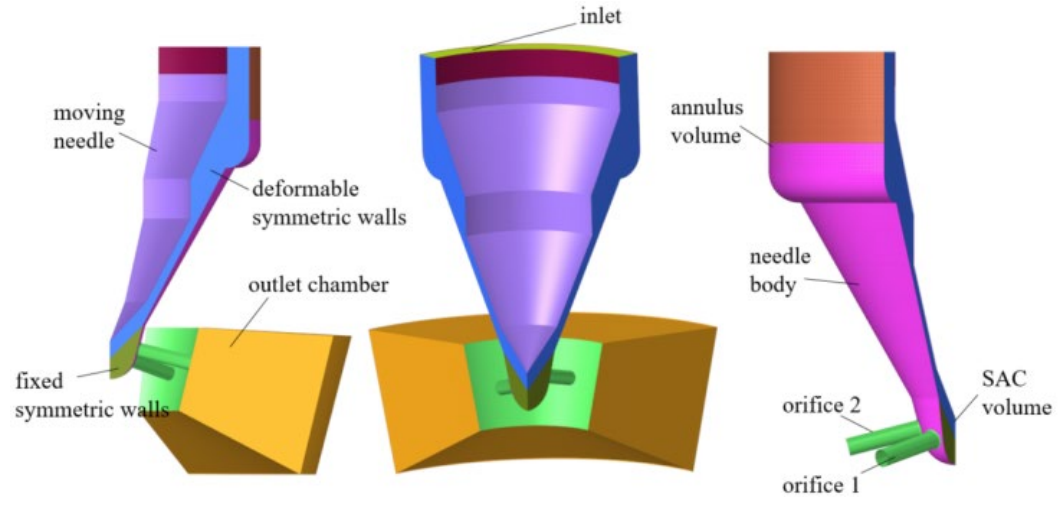

Model validation

Figure 1. Boundary surfaces indicated in the quarter model

The measurement of injection rates within nozzle holes were conducted on a customized test rig based on the theory of the momentum flux. Selected force sensors used for data acquisition 
were one of the integral parts of the set-up. The experiment was conducted at the same working condition of the numerical simulation. Validation was carried out by comparing experimental and simulation injection rates at the exit of the orifices; the comparisons are presented in Figure 2.

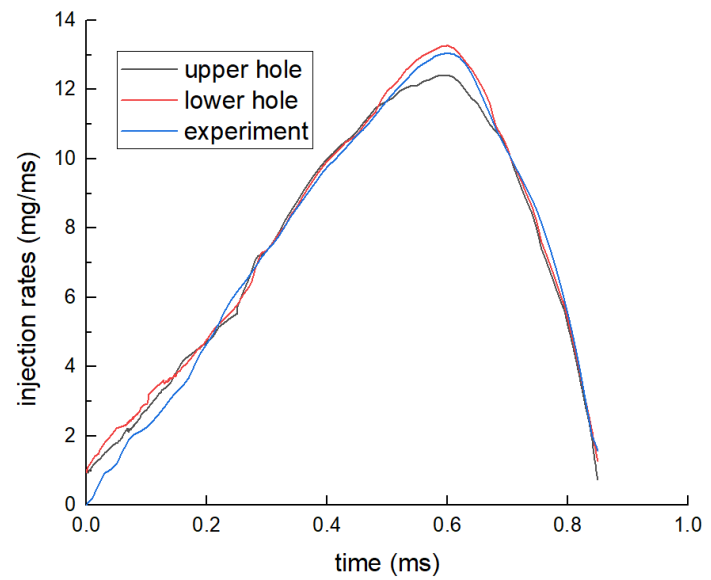

Figure 2. Comparison between experimental and numerical results

\section{Results and Discussion}

The evolution of vapor phase with the time is presented in Fig. 3. It could be seen from the figure that there is a recurrent process of cavitation occurring from $0.065 \mathrm{~ms}$ to $0.09 \mathrm{~ms}$, located at the inlet of the orifices and the needle seat passage. This recurring phenomenon is caused by the periodic formation of vapor in the needle seat passage area and the strong vortex and shedding in the sac volume. Besides, cavitation appears earlier in the orifice 2 and develops faster as compared to cavitation development in the orifice 1. The cavitation in both the upper and the lower layered holes start from the entrance of the orifice and develop in the upper part of the orifices, extending to the exit of the orifices over time.

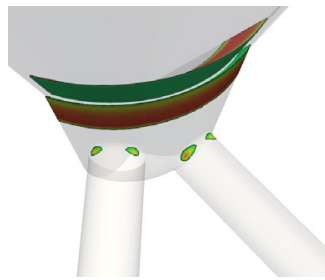

$0.065 \mathrm{~ms}$

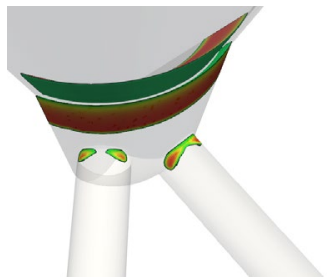

$0.075 \mathrm{~ms}$

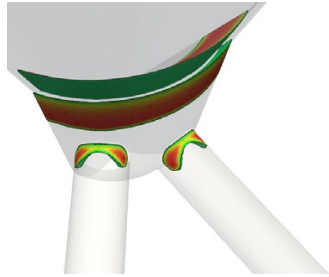

$0.09 \mathrm{~ms}$

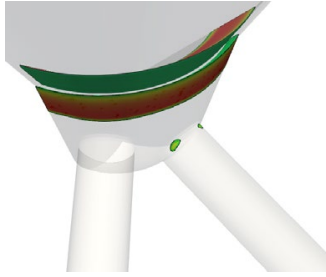

$0.068 \mathrm{~ms}$

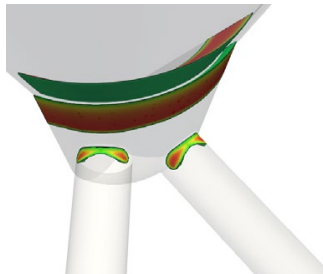

$0.079 \mathrm{~ms}$

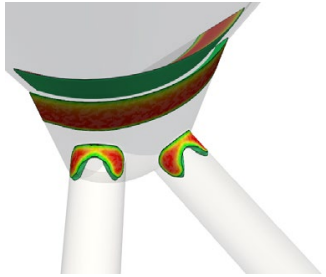

$0.095 \mathrm{~ms}$

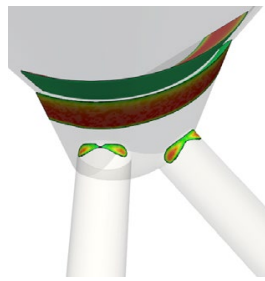

$0.073 \mathrm{~ms}$

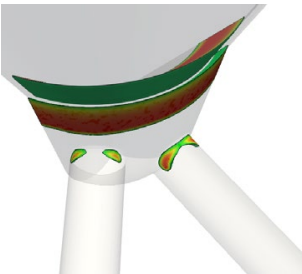

$0.082 \mathrm{~ms}$

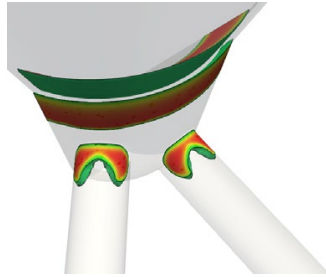

$0.1 \mathrm{~ms}$

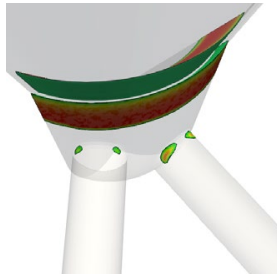

$0.074 \mathrm{~ms}$

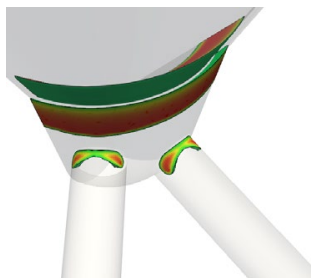

$0.085 \mathrm{~ms}$

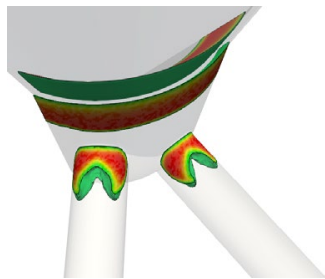

$0.106 \mathrm{~ms}$ 


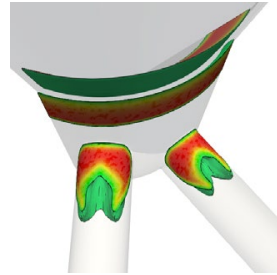

$0.115 \mathrm{~ms}$

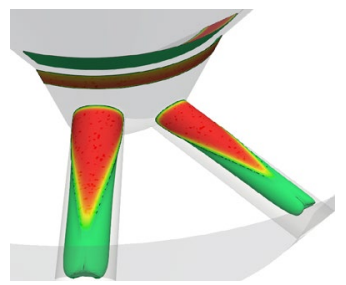

$0.147 \mathrm{~ms}$

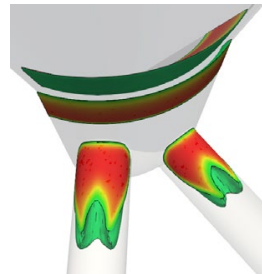

$0.125 \mathrm{~ms}$

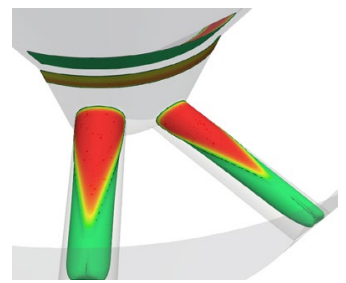

$0.154 \mathrm{~ms}$

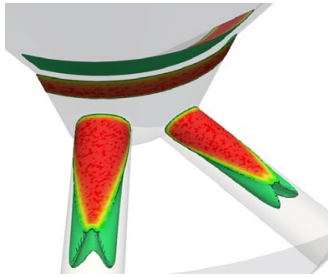

$0.135 \mathrm{~ms}$

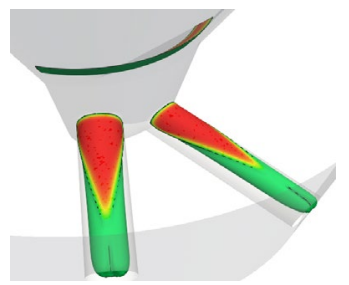

$0.23 \mathrm{~ms}$

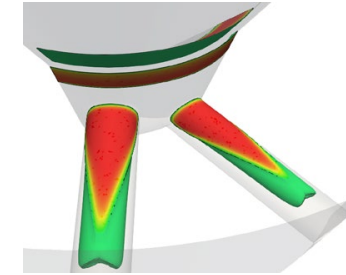

$0.143 \mathrm{~ms}$

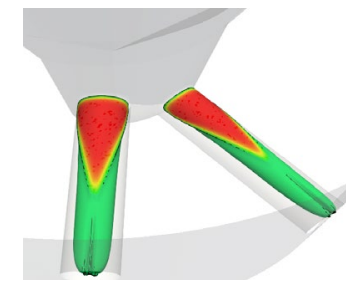

$0.285 \mathrm{~ms}$

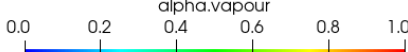

Figure 3. Vapour volume with the time (iso-surface from 0.4 to 1 )

The 3D flow streamlines indicating the vapor and vortex shedding are visualized in the Figure 4. Three small scale vortices and one large scale vortex are emerged with the evolution of injection time, identified as $V_{1}$ to $V_{4}$ respectively. The core of $V_{4}$ is located at the bottom of the SAC, where the fuel jets injected from the upper orifice and lower orifice collide and interact with each other. The vortex structure $\mathrm{V}_{3}$ located before the entrance of the orifices reveals the recurring process of fuel accelerating and decelerating in the needle seat passage area, leading to the regeneration of sheet cavitation. The strong recirculation structures in the orifice 1 and orifice 2 produce the coherent vortex structures at the upper part of the orifices, and dissipate at the time of $140 \mu \mathrm{s}$.
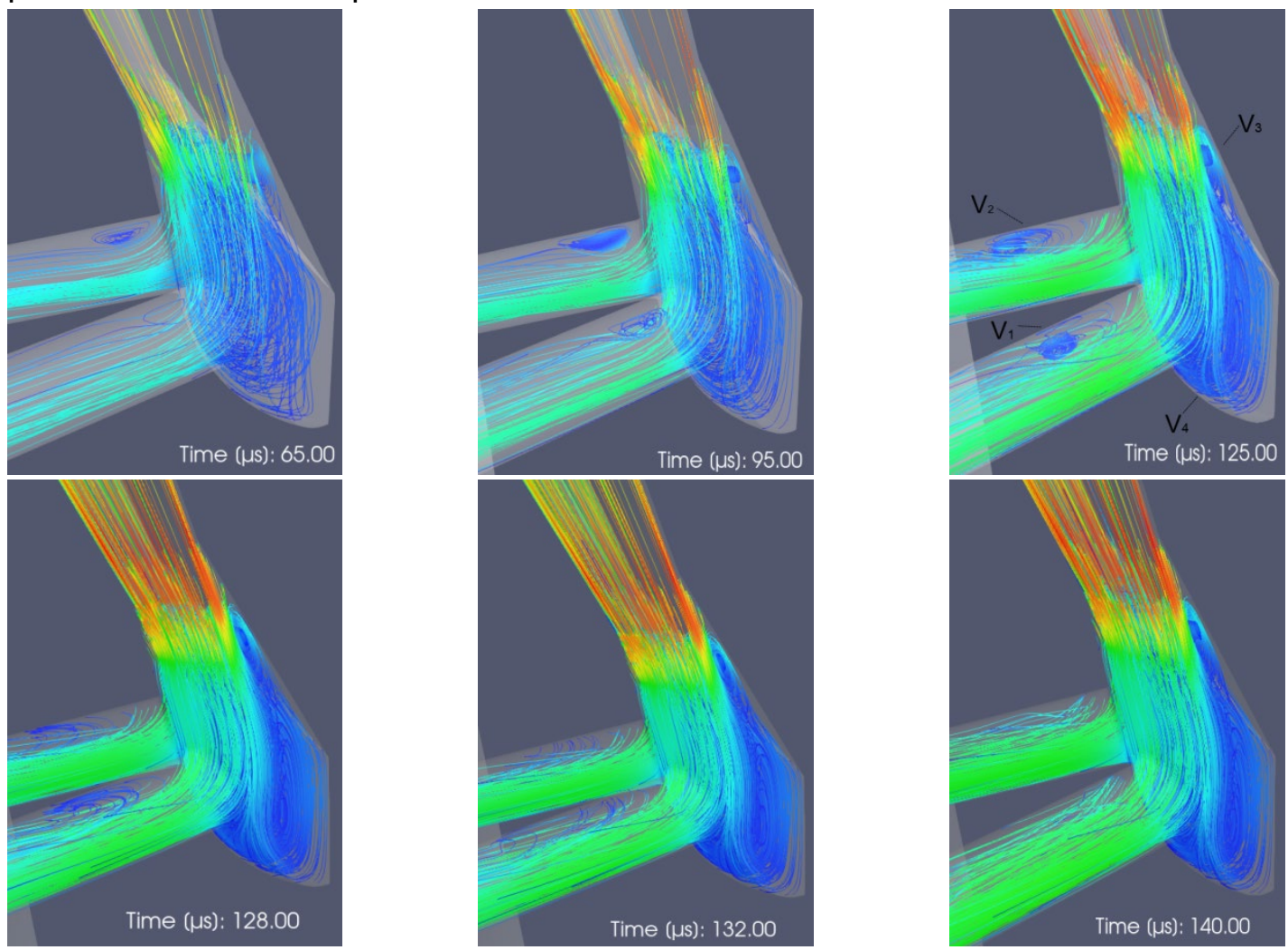

Figure 4. Streamlines with the time 
Figure 5 and Figure 6 present the snapshots of transient velocity magnitude and pressure field. As shown from Figure 3 to Figure 6, cavitation occupies a relative larger area in the orifice 2 than the orifice 1. Furthermore, the acuteness of the orifice 2 means that cavitation development at their entry sections will be more developed than those at the less acute orifice 1.

Orifice1
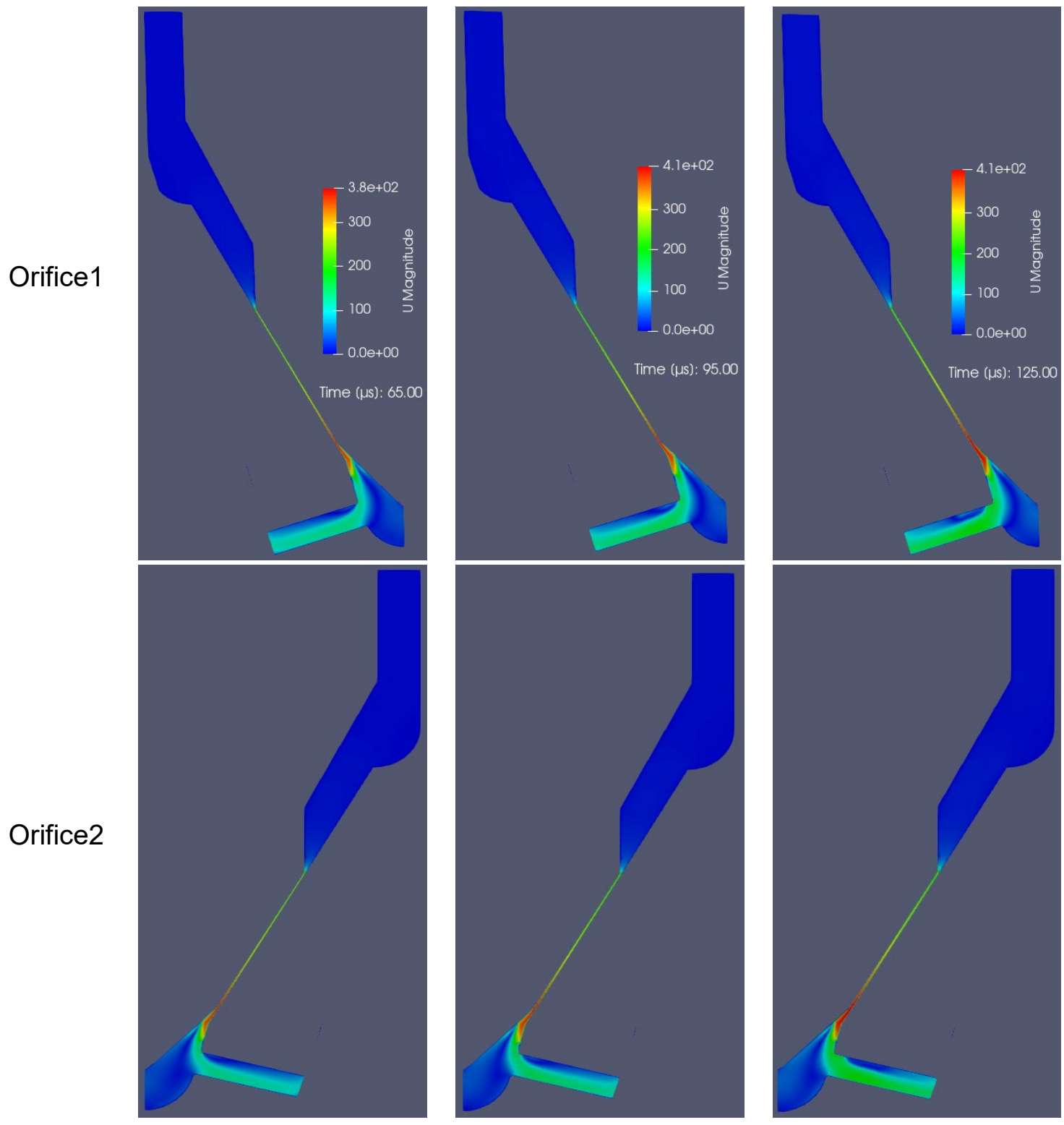

Figure 5. Velocity slices of the upper layered orifice and lower layered orifice
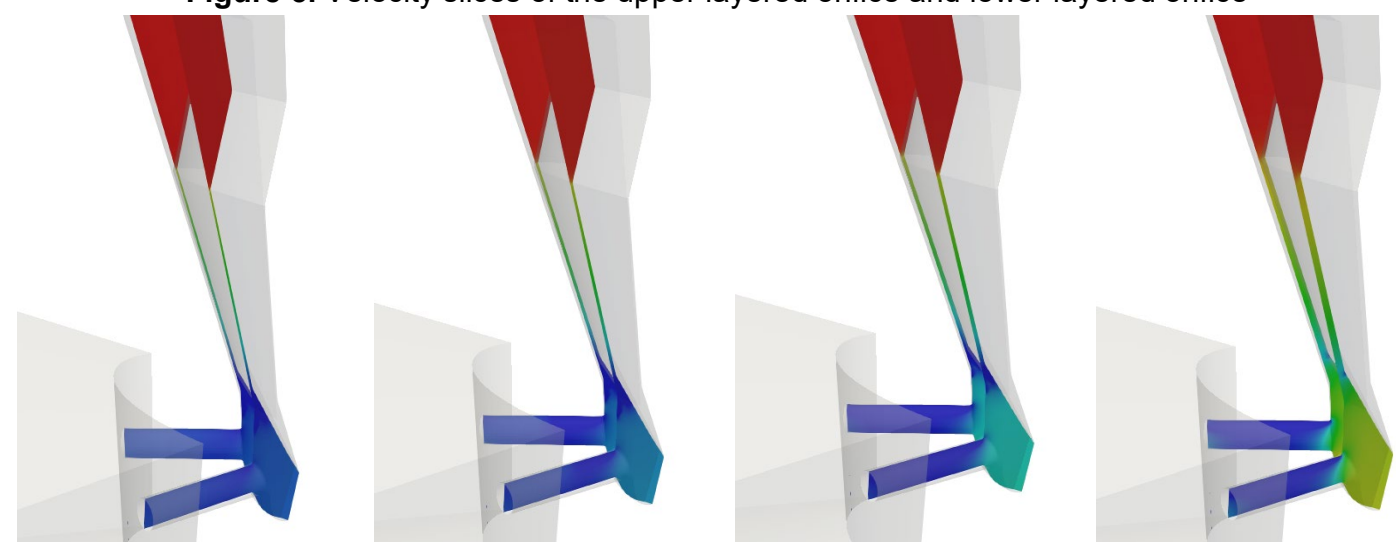


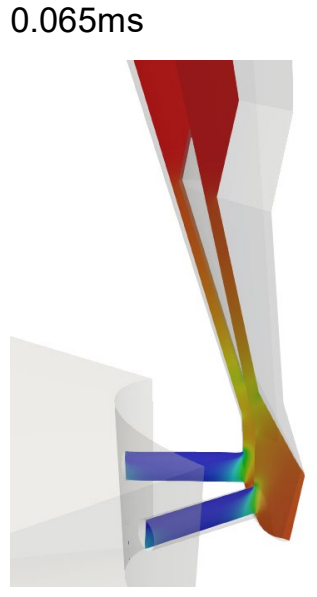

$0.410 \mathrm{~ms}$
$0.125 \mathrm{~ms}$

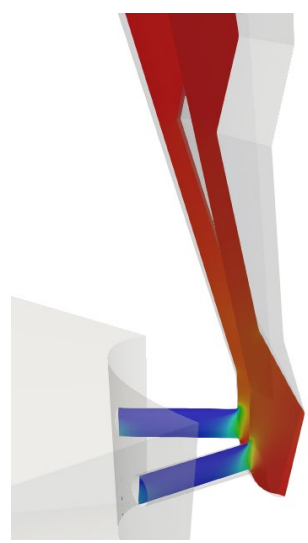

$0.600 \mathrm{~ms}$
$0.190 \mathrm{~ms}$

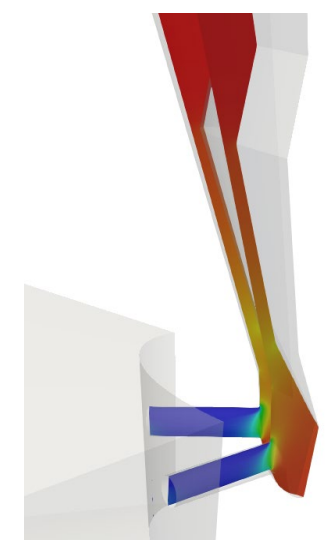

$0.640 \mathrm{~ms}$
$0.300 \mathrm{~ms}$

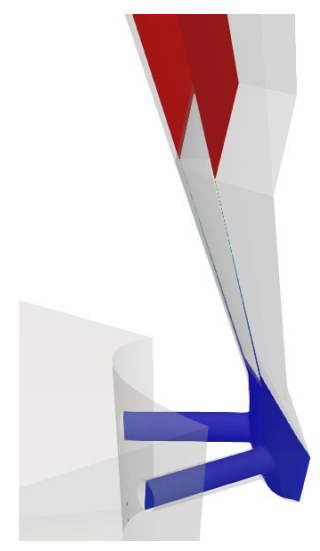

$0.860 \mathrm{~ms}$

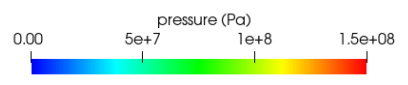

Figure 6. Pressure slices with the time

\section{Conclusion}

Numerical simulation within a double-layer nozzles using the neat PODE was conducted in the study. The following conclusion were made:

1) Due to the different location of the double layered orifices, the acuteness of the upper layer orifice is conductive to the formation of a higher degree of cavitation development in them.

2) The flow re-circulation occurred in both the upper part of the orifices and SAC volume specify two different vapor-vortex shedding process.

3 ) The cycle fuel injection quantities of the lower orifice is higher than the upper one by $6 \%$.

\section{Acknowledgments}

This work was supported by the National Natural Science Foundation of China (No. 51476072), and the China Scholarship Council (CSC No. 201808320261). The authors would also like to appreciate the help from flucon fluid control $\mathrm{GmbH}$ for providing the properties of the fuel and betz-GmbH for measuring the parameters of the nozzle.

\section{References}

[1] C. Arcoumanis, M. Gavaises, J. M. Nouri, E. Abdul-Wahab, and R. W. Horrocks, "Analysis of the flow in the nozzle of a vertical multi-hole diesel engine injector," in SAE Technical Papers, 1998.

[2] Z. He, H. Zhou, L. Duan, M. Xu, Z. Chen, and T. Cao, "Effects of nozzle geometries and needle lift on steadier string cavitation and larger spray angle in common rail diesel injector," Int. J. Engine Res., 2020.

[3] M. G. Mithun, P. Koukouvinis, and M. Gavaises, "Numerical simulation of cavitation and atomization using a fully compressible three-phase model," Phys. Rev. Fluids, vol. 3, no. 6, pp. 0-3, 2018.

[4] L. Zhang, Z. He, W. Guan, Q. Wang, and S. Som, "Simulations on the cavitating flow and corresponding risk of erosion in diesel injector nozzles with double array holes," Int. J. Heat Mass Transf., vol. 124, pp. 900-911, 2018.

[5] N. Kyriazis, P. Koukouvinis, and M. Gavaises, "Numerical investigation of bubble dynamics using tabulated data," Int. J. Multiph. Flow, vol. 93, pp. 158-177, 2017.

[6] P. Koukouvinis, M. Gavaises, J. Li, and L. Wang, "Large Eddy Simulation of Diesel injector including cavitation effects and correlation to erosion damage," Fuel, vol. 175, pp. 26-39, 2016. 
[7] E. Gomez Santos, J. Shi, M. Gavaises, C. Soteriou, M. Winterbourn, and W. Bauer, "Investigation of cavitation and air entrainment during pilot injection in real-size multi-hole diesel nozzles," Fuel, vol. 263, no. November 2019, p. 116746, 2020.

[8] O. Coutier-Delgosha, R. Fortes-Patella, and J. L. Reboud, "Evaluation of the Turbulence Model Influence on the Numerical Simulations of Unsteady Cavitation," J. Fluids Eng., vol. 125, no. 1, p. 38, 2003.

[9] P. Koukouvinis, H. Naseri, and M. Gavaises, "Performance of turbulence and cavitation models in prediction of incipient and developed cavitation," Int. J. Engine Res., vol. 18, no. 4, pp. 333-350, 2017.

[10] M. Gold et al., "Simulation and Measurement of Transient Fluid Phenomena within Diesel Injection," SAE Tech. Pap. Ser., vol. 1, pp. 0-3, 2019.

[11] M. J. Ivings, D. M. Causon, and E. F. Toro, "On Riemann solvers for compressible liquids," Int. J. Numer. Methods Fluids, vol. 28, no. 3, pp. 395-418, 1998.

[12] A. Kulkarni et al., "A Force Field for Poly(oxymethylene) Dimethyl Ethers (OME n)," J. Chem. Theory Comput., vol. 16, no. 4, pp. 2517-2528, 2020. 